\title{
Improving the reproducibility of science
}

In a series of brief articles named Reproducibility Notes, we will highlight topics related to produce robust, effective and reproducible science.

\author{
By Leonhard Held and Simon Schwab \\ Center for Reproducible Science \\ University of Zurich
}

We all love to see science as a fact-producing machinery. However, the lack of reproducibility of claims of new discoveries has caused growing concerns in many scientific communities [1] and beyond. Ultimately, a previously established empirical result can even lose the status as "scientific knowledge" if it does not stand the test of reproducibility over time.

Reproducibility is one of the main principles of the scientific method: can we confirm the result of a study in an independent investigation? This confirmation can take place to various degrees and three types of reproducibility have been identified [2]. First, a study dataset is reanalyzed to verify the results; this is often referred to as computational or methods reproducibility. Second, a new study with new data and the same research question is conducted, known as a result reproducibility or replicability. Ideally, a methods section of a research paper provides precise information about what has been done with the data. However, in practice, verifying research results is more challenging than one might expect, even the simplest statistical tests are sometimes not fully specified. Finally, inferential reproducibility refers to the ultimate goal that different scientists analysing the same dataset should come to similar conclusions. Lack of inferential reproducibility is related to the concept of "researcher degrees of freedom", the inherent flexibility in the design and analysis of a scientific study.

Concerted replication efforts in the last years have revealed that a number of scientific findings could not be replicated. For example, a large portion of one hundred replication studies conducted in psychology produced substantially weaker evidence for the original findings: only a third of the studies have been significant and the average replication effect size was only half of the original effect size. Does this mean that psychology has less rigorous standards than other disciplines? They just may be among the first who systematically investigated the issue. More recent replication attempts were likewise sobering in biomedicine, economics, and the social sciences. However, these replication attempts have also been criticised. For example, there is growing concern about the standard practice to assess the success of a replication study based on simple significance thresholding. Thus, an important part in the growing field of meta-research is the development of novel methods to better define and assess replication success in different scientific disciplines.

The causes of irreproducible research are manyfold, but under-powered studies, post-hoc generation of Hypotheses After the Results are Known (HARKing), p-hacking, and publication bias have been listed as the "four horsemen of the reproducibility crisis" [3]. However, the problems go even further when looking at the speed and quantity "significant" results are being generated today in order to strive for a successful career in science. 
Improving reproducibility requires a broad set of actions [4]. The teaching and application of good research practices in the design, execution, and analysis of studies, especially for early-career researchers. Study registration, analysis plans, and promoting team science are highly relevant as well. A set of new technologies support the organization and documentation of research projects, most prominent the Open Science Framework (www.osf.io). Electronic notebooks can reproduce a research paper's results and statistics, including data figures and tables.

Better statistics plays a central role in improving the reproducibility of science. Statisticians and data scientists should be among those who provide guidance and solutions to tackle the problems of irreproducible research. However, our community seems to be self-absorbed in "statistics wars", as illustrated by the ongoing controversy about p-values and significance tests. Undoubtedly, science would proceed faster if statistical methods are better aligned with scientific needs and practice [5]. The aim of Reproducibility Notes is to give researchers easy access to the statistical tools they need to produce robust, effective and reproducible science. To achieve this, we will carefully highlight topics relevant to the reproducibility of research in future issues of this magazine.

\section{References}

1. Baker, M. (2016) 1,500 scientists lift the lid on reproducibility. Nature, 533, 452-454.

2. Goodman, S. N., Fanelli, D., Ioannidis, J. P. A. (2016) What does research reproducibility mean? Sci. Transl. Med., 8, 341ps12.

3. Bishop, D. (2019) Rein in the four horsemen of irreproducibility. Nature, 568, 435.

4. Munafò, M. R. et al. (2017) A manifesto for reproducible science. Nature Human Behaviour, 1, 0021.

5. Goodman, S. N. (2016) Aligning statistical and scientific reasoning. Science, 352, 1180-1181. 